\title{
The Impact of Disparities in Social Determinants of Health on Hospitalization Rates for Patients with COVID-19 in Michigan (USA)
}

\author{
Megan McCrohan ${ }^{1, *}$ \\ Linnea Nierenberg ${ }^{1, *}$ \\ Patrick Karabon ${ }^{2}$ \\ Tracy Wunderlich-Barillas ${ }^{2}$ \\ Alexandra Halalau (iD) 1,3 \\ 'Oakland University William Beaumont \\ School of Medicine, Rochester, MI, USA; \\ ${ }^{2}$ Office of Research, Oakland University \\ William Beaumont School of Medicine, \\ Rochester, MI, USA; ${ }^{3}$ Internal Medicine \\ Department, Beaumont Health, Royal \\ Oak, MI, USA
}

*These authors contributed equally to this work
Correspondence: Linnea Nierenberg Email nierenberglinnea@gmail.com
Importance: The COVID-19 pandemic continues to impact the health-care system in the United States and has brought further light on health disparities within it. However, only a few studies have examined hospitalization risk with regard to social determinants of health. Objective: We aimed to identify how health disparities affect hospitalization rates among patients with COVID-19.

Design: This observational study included all individuals diagnosed with COVID-19 from February 25, 2020 to December 31, 2020. Uni- and multivariate analyses were utilized to evaluate associations between demographic data and inpatient versus outpatient status for patients with COVID-19.

Setting: Multicenter ( 8 hospitals), largest size health system in Southeast Michigan, a region highly impacted by the pandemic.

Participants: All outpatients and inpatients with a positive RT-PCR for SARS-CoV-2 on nasopharyngeal swab were included. Exclusion criteria included missing demographic data or status as a non-permanent Michigan resident.

Exposure: Patients who met inclusion and exclusion criteria were divided in 2 groups: outpatients and inpatients.

Main Outcome and Measures: We described the comparative demographics and known disparities associated with hospitalization status.

Results: Of 30,292 individuals who tested positive for SARS-CoV-2, 34.01\% were admitted to the hospital. White or Caucasian race was most prevalent $(57.49 \%)$, and $23.35 \%$ were African-American. The most common ethnicity was non-Hispanic or Latino $(70.48 \%)$. English was the primary language for the majority of patients $(91.60 \%)$. Private insurance holders made up $71.11 \%$ of the sample. Within the hospitalized patients, lower socioeconomic status, African-American race and Hispanic and Latino ethnicity, non-English speaking status, and Medicare and Medicaid were more likely to be admitted to the hospital.

Conclusions and Relevance: Several health disparities were associated with greater rates of hospitalization due to COVID-19. Addressing these inequalities from an individual to system level may improve health-care outcomes for those with health disparities and COVID-19.

Keywords: COVID-19, hospitalization, social determinants of health, disparities

\section{Key Points}

Question: Which social determinants of health increase the risk of hospitalization in patients with COVID-19? 
Finding: Lower socioeconomic status as indexed by zip code, African-American race and Hispanic and Latino ethnicity, non-English speaking status, and Medicare and Medicaid patients were all at higher risk of hospitalization in patients with COVID-19.

Meanings: Individual- and population-level health disparities need to be addressed because they lead to serious and tangible consequences for patients as they increase the risk for inpatient admission, which could cause higher morbidity and mortality, due to more severe COVID-19 disease.

\section{Introduction}

The coronavirus disease 2019 (COVID-19) pandemic, continues to impact health-care systems across the United States as it continues to require hospitalization for many. ${ }^{1}$ Clinical predisposing factors for hospitalization including: older age, male gender, obesity, COVID-19 Risk of Complications Score have been reported in southeast Michigan, a region significantly impacted by the pandemic. $^{2,3}$

Ethnic and racial minorities, poverty, and low education levels, ${ }^{4}$ poverty and black race, ${ }^{5-9}$ non-Hispanic black race, ${ }^{7-10}$ public insurance use (ie, Medicare or Medicaid $)^{5,6,11}$ have been reported as health-care disparities having high hospitalization rates.

Our study aimed to assess the impact of multiple social determinants of health on the rates of hospitalization for patients with COVID-19 in southeast Michigan specifically.

\section{Methods}

We conducted an observational study at the largest size healthcare system ( 8 hospitals) in southeast Michigan from February 25, 2020 to December 31, 2020. We included all outpatients and inpatients who were diagnosed with SARS-CoV2 infection by a positive RT-PCR on nasopharyngeal swab. Patients for whom demographic data were missing and patients who were not permanent Michigan residents were excluded from analysis. Zip-code-level data from the United States Census Bureau such as rate of unemployment/use of public transportation/percentage of food stamp use, were used as proxies for economic and employment status as individual-level data was not available from the electronic health record. The zip-code-level data was eventually matched with the individual patient-level data. Univariate and multivariate analysis were used to determine any hospitalization correlates. Assumptions of all models were adequately met. $\mathrm{P}$ values of less than 0.05 were considered statistically significant. Given the large sample size and retrospective nature of the study, it was not feasible to obtain consent from individual patients. The protocol for the study was reviewed and approved by the Beaumont Health Institutional Review Board IRB (\#2020-209).

\section{Results}

Of 30,292 individuals who tested positive for SARS-CoV $-2,10,303(34.01 \%)$ were admitted to the hospital at least once. Approximately half of the patients $(57.49 \%)$ were white or Caucasian and one-quarter (23.35\%) were Black or African-American. The most common ethnicity was non-Hispanic or Latino representing $70.48 \%$ of the total patients. The majority of patients $(91.60 \%)$ spoke English as their primary language. Of the total patients included, $71.11 \%$ had private insurance. These demographics are reflective of the local total population. The remaining descriptive results can be found in Table 1 .

In the univariate analysis, Black or African American patients had $64 \%$ greater odds of admission than White or Caucasian patients (OR: 1.64; CI: 1.55, 1.74). Arabic or Middle Eastern patients had 25\% lower odds of admission than non-Hispanic or Latino patients (OR: 0.75; CI: 0.69-0.81). Non-English speakers had $61 \%$ greater odds of admission than English speakers (OR: 1.61; CI: 1.47, 1.74). Medicaid patients had 2.05-fold greater odds of admission than private insurance patients (OR: 2.05; CI: 1.78, 2.36), and Medicare patients had 4.16-fold greater odds of admission than private insurance patients (OR: 4.16; CI: 3.93, 4.40). On average, patients who lived in zip codes with higher unemployment rates, usage of public transportation, higher percentage of service workers, rates of poverty, and lower rates of white collar professions and median income, were more likely to be hospitalized (all $\mathrm{p}<0.0001$ ).

In the multivariate analysis, independent hospitalization correlates were: Black or African American patients (AOR: 1.83; CI: 1.70, 1.93), Hispanic or Latino patients (AOR: 1.49; CI: 1.27, 1.75), non-English speaking patients (AOR: 1.53; CI: 1.36, 1.71), Medicaid patients (AOR: 1.49; CI: 1.27, 1.74), Medicare patients (AOR: $1.41 ; 1.31,1.53$ ), disabled patients (AOR: 2.23; CI: 1.97, 2.51), unemployed patients (AOR: 1.46, CI: $1.35,1.59$ ). For all reported AOR above, the p value was $<0.0001$. The remaining multivariate results can be found in Table 2 . 
Table I Demographic Characteristics of Patients Testing Positive for SARS-CoV2 Infection from February to December 2020 (N = 30,292) ( $p$ values Were Resulted Through a Chi-Square Test)

\begin{tabular}{|c|c|c|c|c|}
\hline \multirow[t]{2}{*}{ Demographic Characteristics } & Total Patients & Admitted & Not Admitted & \multirow[t]{2}{*}{ P-value } \\
\hline & $\mathbf{N}=30,292$ & $\mathbf{N}=10,303$ & $N=19,989$ & \\
\hline \multicolumn{5}{|l|}{ Age of Patient (Years) $(n=30,291)$} \\
\hline Mean (Standard Deviation) & $53.52(20.11)$ & $63.54(17.77)$ & $48.31(19.15)$ & $<0.0001$ \\
\hline \multicolumn{5}{|l|}{ Body Mass Index (BMI) $(n=15,680)$} \\
\hline Mean (Standard Deviation) & $31.35(8.49)$ & $31.65(8.65)$ & $30.78(7.68)$ & $<0.0001$ \\
\hline \multicolumn{5}{|c|}{ Unemployment Rate of ZIP Code $(\%)(n=29,930)$} \\
\hline Mean (Standard Deviation) & $6.73 \%(4.43 \%)$ & $7.43 \%(4.79 \%)$ & $6.36 \%(4.19 \%)$ & $<0.0001$ \\
\hline \multicolumn{5}{|c|}{$\begin{array}{l}\text { Percent of ZIP Code Taking Public Transportation to Work (\%) } \\
(n=29,930)\end{array}$} \\
\hline Mean (Standard Deviation) & $1.48 \%(2.43 \%)$ & $1.80 \%(2.71 \%)$ & $1.32 \%(2.26 \%)$ & $<0.0001$ \\
\hline \multicolumn{5}{|c|}{$\begin{array}{l}\text { Percent of ZIP Code Working in White Collar Profession (\%) (n } \\
=29,930)\end{array}$} \\
\hline Mean (Standard Deviation) & $37.89 \%(14.32 \%)$ & $35.70 \%(13.85 \%)$ & $39.03 \%(14.43 \%)$ & $<0.0001$ \\
\hline \multicolumn{5}{|c|}{$\begin{array}{l}\text { Percent of ZIP Code Working in Service Profession (\%) } \\
(\mathrm{n}=29,930)\end{array}$} \\
\hline Mean (Standard Deviation) & $17.51 \%(5.50 \%)$ & $18.40 \%(5.59 \%)$ & $17.04 \%(5.40 \%)$ & $<0.0001$ \\
\hline \multicolumn{5}{|c|}{ Median Income of ZIP Code $(\$)(n=29,929)$} \\
\hline \multirow[t]{2}{*}{ Mean (Standard Deviation) } & $\$ 65,360.97$ & $\$ 60,764.35$ & $\$ 67,745.13$ & $<0.0001$ \\
\hline & $(\$ 27,067.02)$ & $(\$ 25,592.06)$ & $(\$ 27,502.70)$ & \\
\hline \multicolumn{5}{|c|}{ Percent of ZIP Code on Food Stamps/SNAP $(\%)(n=29,929)$} \\
\hline Mean (Standard Deviation) & $15.12 \%(\mid 2.71 \%)$ & $17.28 \%(13.28 \%)$ & $14.00 \%(12.25 \%)$ & $<0.0001$ \\
\hline \multicolumn{5}{|l|}{ Poverty Rate of ZIP Code $(\%)(n=29,929)$} \\
\hline Mean (Standard Deviation) & $11.85 \%(10.39 \%)$ & $13.39 \%(10.84 \%)$ & $11.05 \%(10.06 \%)$ & $<0.0001$ \\
\hline \multicolumn{5}{|l|}{ Biological Sex of Patient $(n=30,292)$} \\
\hline Female & $16,454(54.32 \%)$ & $5184(50.38 \%)$ & $\mathrm{II}, 250(56.32 \%)$ & $<0.0001$ \\
\hline Male & $|3,83|(45.66 \%)$ & $5106(49.62 \%)$ & $8717(43.64 \%)$ & \\
\hline Unknown & $7(0.02 \%)$ & $0(0.00 \%)$ & $7(0.04 \%)$ & $N / A^{\prime}$ \\
\hline \multicolumn{5}{|l|}{ Race of Patient $(n=30,292)$} \\
\hline American Indian or Alaska Native & $74(0.24 \%)$ & $33(0.32 \%)$ & $4 \mid(0.21 \%)$ & $<0.0001$ \\
\hline Asian & 599 (1.98\%) & 208 (2.02\%) & $390(1.95 \%)$ & \\
\hline Black or African American & $7072(23.35 \%)$ & 3308 (32.15\%) & $3758(18.82 \%)$ & \\
\hline Native American or Pacific Islander & $29(0.10 \%)$ & $5(0.05 \%)$ & $24(0.12 \%)$ & \\
\hline Other & $2434(8.04 \%)$ & $659(6.40 \%)$ & I 773 (8.88\%) & \\
\hline White or Caucasian & $17,4 \mid 6(57.49 \%)$ & $6071(59.00 \%)$ & II,328 (56.73\%) & \\
\hline Unknown & $2668(8.81 \%)$ & $6(0.06 \%)$ & $2653(13.29 \%)$ & \\
\hline \multicolumn{5}{|l|}{ Ethnicity of Patient $(n=30,292)$} \\
\hline Arabic or Middle Eastern & $3180(10.50 \%)$ & $1042(10.13 \%)$ & $2138(10.71 \%)$ & $<0.0001$ \\
\hline Hispanic or Latino & $882(2.91 \%)$ & 338 (3.28\%) & $543(2.72 \%)$ & \\
\hline Not Hispanic or Latino & $21,349(70.48 \%)$ & 8404 (81.67\%) & $12,923(64.72 \%)$ & \\
\hline Other & $1395(4.61 \%)$ & 406 (3.95\%) & $986(4.94 \%)$ & \\
\hline Unknown & $3486(11.51 \%)$ & $100(0.97 \%)$ & 3377 (16.91\%) & \\
\hline
\end{tabular}

(Continued) 
Table I (Continued).

\begin{tabular}{|c|c|c|c|c|}
\hline \multirow[t]{2}{*}{ Demographic Characteristics } & Total Patients & Admitted & Not Admitted & \multirow[t]{2}{*}{ P-value } \\
\hline & $\mathbf{N}=\mathbf{3 0 , 2 9 2}$ & $\mathbf{N}=10,303$ & $N=19,989$ & \\
\hline \multicolumn{5}{|l|}{ Marital Status $(n=30,292)$} \\
\hline Divorced & 2247 (7.42\%) & $986(9.58 \%)$ & $1259(6.31 \%)$ & $<0.0001$ \\
\hline Married & $13,182(43.52 \%)$ & 4767 (46.33\%) & $84 I I(42.12 \%)$ & \\
\hline Separated & $245(0.81 \%)$ & $114(1.11 \%)$ & $|3|(0.66 \%)$ & \\
\hline Single & $9522(31.43 \%)$ & $2711(26.35 \%)$ & $6810(34.11 \%)$ & \\
\hline Widowed & 2608 (8.61\%) & $1565(15.21 \%)$ & $1025(5.13 \%)$ & \\
\hline Unknown & $2488(8.21 \%)$ & $147(1.43 \%)$ & $2331(11.67 \%)$ & \\
\hline \multicolumn{5}{|l|}{ English Language Speaker $(n=30,292)$} \\
\hline Yes & $27,746(91.60 \%)$ & $9165(89.07 \%)$ & $18,548(92.89 \%)$ & $<0.0001$ \\
\hline No & $2546(8.40 \%)$ & 1125 (10.93\%) & $1419(7.11 \%)$ & \\
\hline \multicolumn{5}{|l|}{ Primary Payor $(n=30,292)$} \\
\hline Private Insurance & $21,54 \mid(71.11 \%)$ & 5547 (53.91\%) & I5,977 (80.02\%) & $<0.0001$ \\
\hline Uninsured & $640(2.11 \%)$ & $122(1.19 \%)$ & 517 (2.59\%) & \\
\hline Medicaid & $821(2.71 \%)$ & $341(3.31 \%)$ & $480(2.40 \%)$ & \\
\hline Medicare & $7214(23.81 \%)$ & $4253(41.33 \%)$ & $2944(14.74 \%)$ & \\
\hline Tricare/VA & $76(0.25 \%)$ & $27(0.26 \%)$ & $49(0.25 \%)$ & \\
\hline \multicolumn{5}{|c|}{ Has Primary Care Physician (PCP) $(n=30,292)$} \\
\hline Yes & $21,327(70.40 \%)$ & 7746 (75.28\%) & $13,560(67.91 \%)$ & $<0.0001$ \\
\hline No & 8965 (29.60\%) & $2544(24.72 \%)$ & 6407 (32.09\%) & \\
\hline
\end{tabular}

Note: 'Unknown gender was not further analyzed due to no unknown gender patients being admitted.

\section{Discussion}

Our study found that several social determinants of health put patients at risk for hospitalization during COVID-19 infection, including lower socioeconomic status, indexed by zip code and employment status, race, ethnicity, English as a second language, and public insurance. Prior studies have associated similar disparities - such as ethnic and racial minority groups, ${ }^{7-11}$ poverty, ${ }^{5}$ lower education levels, ${ }^{4}$ public insurance coverage ${ }^{5,6,11}$ - with higher hospitalization rates in those infected with COVID-19, yet the topic remains understudied, ${ }^{7-11}$ and only few have focused on the risk of inpatient admission as a primary outcome $e^{4,6,9-11}$ or addressed the breadths of social determinants, as our study has.

These findings using data from Southeastern Michigan's largest size healthcare system suggest that social determinants of health impact what level of care is required for individuals who contract COVID-19. Inpatient care for those of lower socioeconomic status, minority groups, and public insurance users calls not only for greater health-care expense and physical and mental distress of hospitalization to such individuals but shines light on the necessity to address these disparities.
Efforts towards public health education for patients and physicians, acknowledgement of biases and disparities spanning from an individual to public policy level, and further research to better understand social determinants of health may all help to begin to alleviate this gap.

Limitations of this study include relying on a single healthcare system in one geographical region and the inherent limitations of retrospective study design, like unknown confounders and the risk of type 1 error. A small number of patients included in the study had already been admitted to the hospital for non-COVIDrelated reasons and tested positive during the course of their hospitalization; they were not removed from the dataset because it is unclear whether or not the severity of their COVID-19 infection would or would not have resulted in hospital admission. Lastly, another possible limitation is confounding comorbidities associated with social determinants of health that increase the likelihood for necessitating inpatient status. This study aims to broadly identify whether social determinants of health impact risk of hospitalization for patients with COVID-19 infection, such as socioeconomic status, race, ethnicity, and gender. If so, further research is needed to better define and establish 
Table 2 Multivariate Analysis of Patients Testing Positive for SARSCoV2 Infection from February to December $2020(N=30,292)$

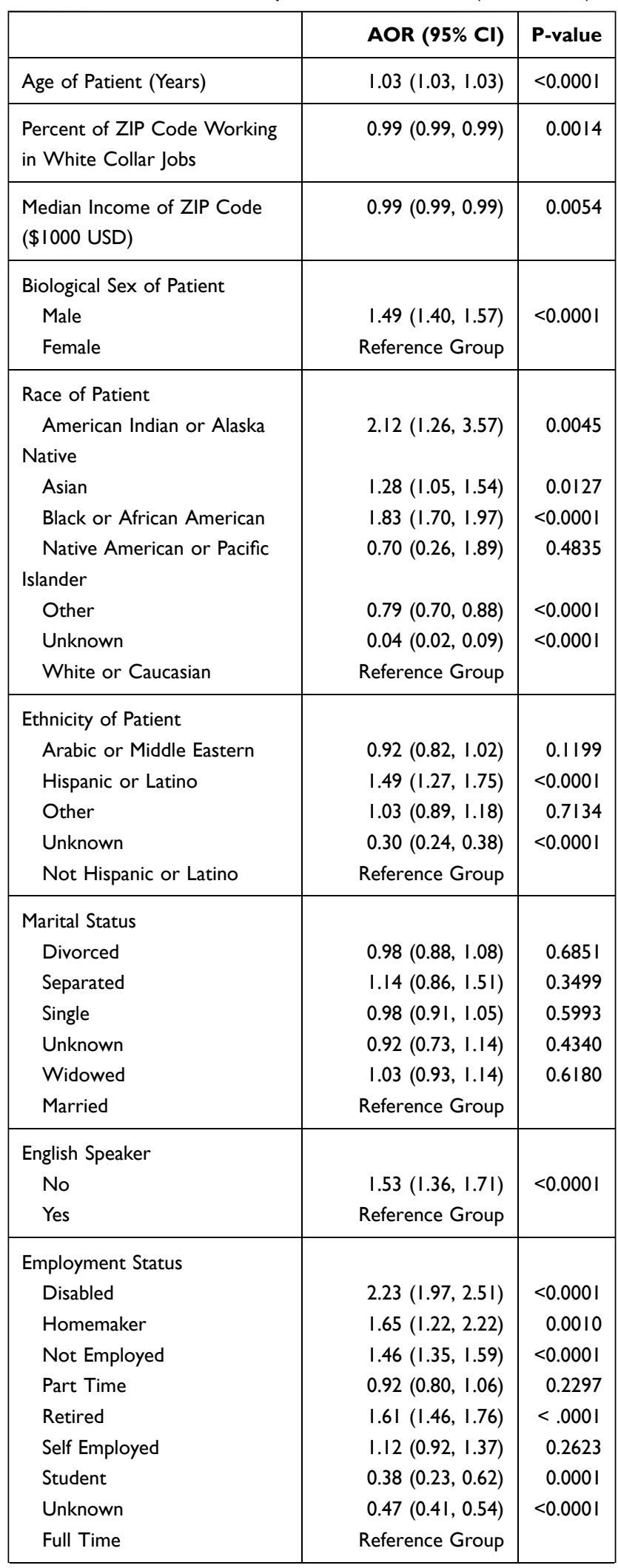

(Continued)
Table 2 (Continued).

\begin{tabular}{|l|r|r|}
\hline & AOR (95\% CI) & P-value \\
\hline Insurance Status & & \\
Medicaid & $I .49(1.27,1.74)$ & $<0.000 \mathrm{I}$ \\
Medicare & $\mathrm{I} .4 \mathrm{I}(\mathrm{I} .3 \mathrm{I}, \mathrm{I} .53)$ & $<0.000 \mathrm{I}$ \\
Tricare/VA & $\mathrm{I} .46(0.84,2.53)$ & 0.1752 \\
Uninsured & $0.8 \mathrm{I}(0.65, \mathrm{I} .02)$ & $0.07 \mathrm{I} 2$ \\
Private Insurance & Reference Group & \\
\hline Primary Care Physician (PCP) & & \\
No & $\mathrm{I} .20(\mathrm{I} .13, \mathrm{I} .28)$ & $<0.000 \mathrm{I}$ \\
Yes & Reference Group & \\
\hline
\end{tabular}

the etiology for these disparities and why individual populations face more serious health outcomes.

\section{Conclusion}

Our study demonstrates that several social determinants of health may put patients at increased risk of hospitalization during COVID-19 infection, including lower socioeconomic status, indexed by zip code and employment status, race, ethnicity, English as a second language, and public insurance. Further research, public health education, and acknowledgement of biases among patients, physicians, policy makers, and health-care systems is necessary to address these disparities in order to decrease risk of hospitalization for patients with COVID-19 who are affected by them.

\section{Data Sharing Statement}

The data used to support the findings of this study are available from the corresponding author upon request.

\section{Ethics Approval}

The study was approved by the Beaumont Health Institutional Review Board. The study was approved under expedited review and the patient consent was waived as the study design was retrospective. The data confidentiality and compliance with the Declaration of Helsinki were maintained.

\section{Funding}

This research did not receive any specific funding.

\section{Disclosure}

Megan McCrohan and Linnea Nierenberg are co-first authors for this study. The authors have no conflicts of interest regarding the publication of this paper. 


\section{References}

1. Moghadas SM, Shoukat A, Fitzpatrick MC, et al. Projecting hospital utilization during the COVID-19 outbreaks in the United States. Proc Natl Acad Sci. 2020;117(16):9122-9126. doi:10.1073/pnas.20040 64117

2. Imam Z, Odish F, Armstrong J, et al. Independent correlates of hospitalization in 2040 patients with COVID-19 at a large hospital system in Michigan, United States. J Gen Intern Med. 2020;35 (8):2516-2517. doi:10.1007/s11606-020-05937-5

3. Halalau A, Imam Z, Karabon P, et al. External validation of a clinical risk score to predict hospital admission and in-hospital mortality in COVID-19 patients. Ann Med. 2021;53(1):78-86. doi:10.1080/07853 890.2020 .1828616

4. Wadhera RK, Wadhera P, Gaba P, et al. Variation in COVID-19 hospitalizations and deaths across New York City Boroughs. JAMA. 2020;323(21):2192-2195. doi:10.1001/jama.2020.7197

5. Muñoz-Price LS, Nattinger AB, Rivera F, et al. Racial disparities in incidence and outcomes among patients with COVID-19. JAMA Network Open. 2020;3(9):e2021892. doi:10.1001/jamanetworkopen.20 20.21892
6. Price-Haywood EG, Burton J, Fort D, Seoane L. Hospitalization and mortality among black patients and white patients with Covid- 19 . $N$ Engl $J$ Med. 2020;382(26):2534-2543. doi:10.1056/NEJMsa 2011686

7. Seldon TM, Berdahl TA. COVID-19 and racial/ethnic disparities in health risk, employment, and household composition. Health Affiars. 2020;39(9):1624-1632. doi:10.1377/hlthaff.2020.00897

8. Mackey K, Ayers CK, Kondo KK, et al. Racial and ethnic disparities in COVID-19-related infections, hospitalizations, and deaths. Ann Intern Med. 2021;174(3):362-373. doi:10.7326/M20-6306

9. Krishnamoorthy G, Arsene C, Jena N, et al. Racial disparities in COVID-19 hospitalizations do not lead to disparities in outcomes. Public Health. 2021;190:93-98. doi:10.1016/j.puhe.2020.11.021

10. Ko JY, Danielson ML, Town M, et al. Risk factors for COVID-19associated hospitalization: COVID-19-associated hospitalization surveillance network and behavioral risk factor surveillance system. Clin Infect Dis. 2020;ciaa1419. doi:10.1093/cid/ciaa1419

11. Killerby ME, Link-Gelles R, Haight SC, et al. Characteristics associated with hospitalization among patients with COVID-19 Metropolitan Atlanta, Georgia, March-April 2020. Morb Mortal Wkly Rep. 2020;69:790-794. doi:10.15585/mmwr.mm6925e1

\section{Publish your work in this journal}

The International Journal of General Medicine is an international, peer-reviewed open-access journal that focuses on general and internal medicine, pathogenesis, epidemiology, diagnosis, monitoring and treatment protocols. The journal is characterized by the rapid reporting of reviews, original research and clinical studies across all disease areas. The manuscript management system is completely online and includes a very quick and fair peer-review system, which is all easy to use. Visit http://www.dovepress.com/ testimonials.php to read real quotes from published authors. 